\title{
Mid-infrared photometry of the T Tauri triple system with kernel phase interferometry ${ }^{\star}$
}

\author{
J. Kammerer ${ }^{1,2}$, M. Kasper ${ }^{1}$, M. J. Ireland ${ }^{2}$, R. Köhler ${ }^{3}$, R. Laugier ${ }^{4}$, F. Martinache ${ }^{4}$, R. Siebenmorgen ${ }^{1}$, \\ M. E. van den Ancker ${ }^{1}$, R. van Boekel ${ }^{5}$, T. M. Herbst ${ }^{5}$, E. Pantin ${ }^{6}$, H.-U. Käufl ${ }^{1}$, \\ D. J. M. Petit dit de la Roche ${ }^{1}$, and V. D. Ivanov ${ }^{1}$
}

\author{
${ }^{1}$ European Southern Observatory, Karl-Schwarzschild-Str. 2, 85748 Garching, Germany \\ e-mail: jens.kammerer@eso.org \\ 2 Research School of Astronomy \& Astrophysics, Australian National University, Canberra, ACT 2611, Australia \\ 3 University of Vienna, Department of Astrophysics, Türkenschanzstr. 17 (Sternwarte), 1180 Vienna, Austria \\ 4 Université Côte d'Azur, Observatoire de la Côte d'Azur, CNRS, Laboratoire Lagrange, Lagrange, France \\ 5 Max Planck-Institut für Astronomie, Königstuhl 17, 69117 Heidelberg, Germany \\ ${ }^{6}$ Laboratoire CEA, IRFU/DAp, AIM, Université Paris-Saclay, Université Paris Diderot, Sorbonne Paris Cité, CNRS, 91191 Gif-sur- \\ Yvette, France
}

Received 8 September 2020 / Accepted 25 November 2020

\section{ABSTRACT}

\begin{abstract}
Context. T Tauri has long been the prototypical young pre-main-sequence star. However, with increasing resolution and sensitivity, $\mathrm{T}$ Tauri has now been decomposed into a triple system with a complex disk and outflow geometry.

Aims. We aim to measure the brightness of all three components of the T Tauri system (T Tau N, T Tau Sa, and T Tau Sb) in the midinfrared in order to obtain photometry around the $\sim 9.7 \mu \mathrm{m}$ silicate feature. This allows us to study their variability and to investigate the distribution of dust and the geometry of circumstellar and circumbinary disks in this complex system.

Methods. We observe the T Tauri system with the Very Large Telescope (VLT)/VISIR-NEAR instrument, performing diffractionlimited imaging in the mid-infrared. With kernel phase interferometry post-processing of the data, and using the astrometric positions of all three components from VLT/SPHERE, we measure the three components' individual brightnesses (including the southern binary at an angular separation down to $\sim 0.2 \lambda / D$ ) and obtain their photometry. In order to validate our methods, we simulate and recover mock data of the T Tauri system using the observed reference point-spread function of HD 27639.

Results. We find that $\mathrm{T}$ Tau $\mathrm{N}$ is rather stable and shows weak silicate emission, while $\mathrm{T}$ Tau Sa is highly variable and shows prominent silicate absorption. T Tau Sb became significantly fainter compared to data from 2004 and 2006, suggesting increased extinction by dust. The precision of our photometry is limited by systematic errors in kernel phase interferometry, which is consistent with previous studies using this technique.

Conclusions. Our results confirm the complex scenario of misaligned disks in the T Tauri system that had been observed previously, and they are in agreement with the recently observed dimming of T Tau Sb in the near-infrared. Our mid-infrared photometry supports the interpretation that $\mathrm{T} \mathrm{Tau} \mathrm{Sb}$ has moved behind the dense region of the Sa-Sb circumbinary disk on its tight orbit around Sa, therefore suffering increased extinction.
\end{abstract}

Key words. stars: variables: $\mathrm{T}$ Tauri, Herbig $\mathrm{Ae} / \mathrm{Be}$ - binaries: close - techniques: high angular resolution

\section{Introduction}

$\mathrm{T}$ Tauri (T Tau) is the historical prototype of a young and accreting low-mass star. As such, it has provided astronomers with many surprise discoveries over the past decades. While $\mathrm{T}$ Tau was initially believed to be a single star surrounded by a circumstellar disk (CSD), from which it accretes matter, Dyck et al. (1982) used near-infrared speckle interferometry to show that it is in fact a binary composed of an optically bright northern component ( T Tau N) and an optically faint southern component (T Tau S). It was suspected that the optical faintness of $\mathrm{T}$ Tau $\mathrm{S}$ is caused by extinction from circumstellar material that hides the southern component behind gas and dust. Indeed, Ghez et al. (1991) found T Tau S to be variable at all near-infrared to mid-infrared wavelengths and concluded that its spectral energy distribution (SED) must be dominated by variable accretion from a CSD. Five years later, the detection of

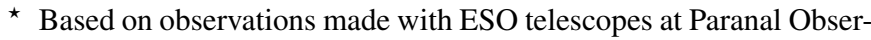
vatory under program ID 60.A-9107(E). perpendicular jets expelled from $\mathrm{T}$ Tau $\mathrm{N}$ and $\mathrm{S}$ supported the theory that $\mathrm{T}$ Tau is composed of not one, but two young and accreting stars (Herbst et al. 1996).

When Koresko (2000) observed T Tau S with the Keck NearIR Camera (NIRC) using speckle interferometry, they found that it is itself a tight binary consisting of $\mathrm{T}$ Tau Sa and $\mathrm{T}$ Tau $\mathrm{Sb}$, and the detection of significant orbital motion of $\mathrm{T}$ Tau $\mathrm{Sa}$ around Sb by Duchêne et al. (2002) implied a high mass ratio between the two southern components. Their mediumresolution spectroscopy further revealed that $\mathrm{T}$ Tau $\mathrm{Sa}$ has a featureless spectrum and that $\mathrm{Sb}$ is an embedded M-type classical T Tauri star (CTTS), suggesting that both southern components are surrounded by dense material. Later, Kasper et al. (2002) and Duchêne et al. (2005) found the CSD of T Tau Sa to be oriented edge-on and attributed the strong optical extinction of $\sim 15 \mathrm{mag}$ around the southern components to circumbinary material (e.g., an Sa-Sb circumbinary disk). Duchêne et al. (2006) identified T Tau Sa to be the most massive component of the triple system, most likely a young Herbig Ae star, from orbital monitoring. 
Table 1. Observing log for program 60.A-9107(E).

\begin{tabular}{lccccccccc}
\hline \hline Start time $(\mathrm{UT})$ & Object & Filter & $\lambda[\mu \mathrm{m}]$ & NDIT & DIT [ms] & $N_{\text {chop }}$ & $\nu_{\text {chop }}[\mathrm{Hz}]$ & $N_{\text {avg }}$ & $N_{\text {good }}$ \\
\hline 02:01:24 & T Tau & NEII & 12.80 & 8 & 25 & 66 & 2.222 & 10 & 10 \\
02:08:18 & T Tau & SIV_1 & 9.81 & 9 & 22 & 75 & 2.273 & 12 & 6 \\
02:16:51 & T Tau & SIV_2 & 10.71 & 9 & 22 & 75 & 2.273 & 12 & 12 \\
02:25:28 & T Tau & ARIII & 8.98 & 6 & 80 & 40 & 0.893 & 15 & 7 \\
03:05:48 & HD27639 & NEII & 12.80 & 8 & 25 & 66 & 2.222 & 4 & 3 \\
03:08:58 & HD27639 & SIV_1 & 9.81 & 9 & 22 & 75 & 2.273 & 4 & 2 \\
03:12:26 & HD27639 & SIV_2 & 10.71 & 9 & 22 & 75 & 2.273 & 8 & 6 \\
$03: 28: 05$ & HD27639 & ARIII & 8.98 & 6 & 80 & 40 & 0.893 & 4 & 2 \\
\hline
\end{tabular}

Notes. All data were taken on December 16, 2019. Reported are the number of exposures per chopping position (NDIT), the exposure time (DIT), the number of chopping cycles $\left(N_{\text {chop }}\right)$, the chopping frequency $\left(v_{\text {chop }}\right)$, the number of frames after averaging $\left(N_{\text {avg }}\right)$, and the number of good frames $\left(N_{\text {good }}\right)$.

Around 2005, the triple system was first resolved in the $\mathrm{N}$ band by two teams. Ratzka et al. (2009) used the Very Large Telescope Interferometer (VLTI) MID-infrared Interferometric instrument (MIDI) to observe T Tau in November 2004. They found that the silicate band at $9.7 \mu \mathrm{m}$ is seen in absorption around both southern components, which confirmed a high extinction by $\mathrm{Sa}-\mathrm{Sb}$ circumbinary material. Moreover, they resolved a small edge-on disk around $\mathrm{T}$ Tau $\mathrm{Sa}$, roughly oriented from north to south. Skemer et al. (2008) used the Multiple Mirror Telescope (MMT) deformable secondary mirror for adaptive opticsassisted imaging in the mid-infrared in November 2006. The high Strehl ratio and the good point-spread function (PSF) stability allowed them to resolve the southern binary at one-third of the classical diffraction limit of a telescope. Their data allowed them to conclude that the material producing the strong silicate absorption toward T Tau S is entirely in front of Sa. Later, van Boekel et al. (2010) measured a very rapid flux increase at $12.8 \mu \mathrm{m}$ of the unresolved T Tau $\mathrm{S}$ binary over just four days, which they attributed to an increased accretion rate. Slower variations could still be well produced by variable extinction, and the most likely scenario is a combination of both processes. Also in 2005, Monnier et al. (2009) observed T Tau in the midinfrared using a technique called segment-tilting, which is a hybrid between aperture masking and kernel phase interferometry, utilizing almost the entire Keck primary mirror while separating the light into multiple non-redundant interferograms. However, their observations were limited by calibration issues caused by bandwidth smearing and the close southern binary could not be properly resolved.

Through orbital monitoring with the Very Large Telescope (VLT) Spectro-Polarimetric High-contrast Exoplanet Research instrument (SPHERE), Köhler et al. (2016) were able to precisely measure the orbits of all three components of the T Tau system, as well as their masses. They found the masses of T Tau $\mathrm{Sa}$ and $\mathrm{Sb}$ to be $\sim 2.12 M_{\odot}$ and $\sim 0.53 M_{\odot}$, respectively, using a mass of $\sim 1.83-2.14 M_{\odot}$ for $\mathrm{T}$ Tau N (Loinard et al. 2007). Recent near-infrared polarimetry suggests an Sa-Sb circumbinary disk (CBD) with a size of $\sim 150$ mas and a position angle of $\sim 30 \mathrm{deg}$ (Yang et al. 2018). This would imply that T Tau Sb is currently moving along its orbit around $\mathrm{Sa}$ through the $\mathrm{Sa}-\mathrm{Sb}$ CBD plane and should therefore suffer noticeable extinction for the first time since its discovery. Indeed, $\mathrm{T}$ Tau $\mathrm{Sb}$ has recently been dimming in the near-infrared (Schaefer et al. 2020), and Köhler \& Kubiak (2020) proposed that this dimming is caused by $\mathrm{Sb}$ passing through the $\mathrm{Sa}-\mathrm{Sb} \mathrm{CBD}$ plane, based on the $J-K$ band photometry of the system.

In this paper, we present new mid-infrared $(\sim 10 \mu \mathrm{m})$ photometry of all three components of the $\mathrm{T}$ Tau system obtained with the VLT Imager and Spectrometer for mid-IR-New Earths in the Alpha Centauri Region instrument (VISIR-NEAR) using kernel phase interferometry. Our observations were conducted in December 2019 when the southern binary was separated by only $\sim 64$ mas, which corresponds to $\sim 0.2 \lambda / D$ at the longest observed wavelengths. Our data allow us to study the brightness of the individual components on and off the $9.7 \mu \mathrm{m}$ silicate feature as well as their variability. The silicate feature is interesting since it contains information about the nature of the circumstellar dust and about the orientation and geometry of the CSDs in this everfascinating system.

\section{Observations}

We observed T Tau with the VISIR-NEAR instrument of the European Southern Observatory's (ESO) VLT at the summit of Cerro Paranal in Chile. VISIR-NEAR is mounted on the Cassegrain focus of Unit Telescope 4 (UT4) and consists of the VISIR instrument (Lagage et al. 2004) plus the NEAR upgrade (Käufl et al. 2018), which enables mid-infrared imaging with extreme adaptive optics. Our observations are part of the VISIR-NEAR science demonstration program 60.A-9107(E), PI M. Kasper, and are summarized in Table 1.

We acquired data for both $\mathrm{T}$ Tau and a PSF reference (HD 27639) in pupil-tracking mode, at wavelengths of $8.98 \mu \mathrm{m}$ (filter name ARIII), $9.81 \mu \mathrm{m}$ (SIV_1), $10.71 \mu \mathrm{m}$ (SIV_2), and $12.80 \mu \mathrm{m}$ (NEII). The sky offset between T Tau and the PSF reference is $\sim 1.3 \mathrm{deg}$, and the difference in average airmass is $\sim 0.04$. Atmospheric turbulence was removed almost entirely by the adaptive optics facility (AOF, Ströbele et al. 2006) of UT4, leaving quasi-static aberrations (such as chromatic aberrations of the filters) as the dominant systematic error. These errors can be identified and calibrated by our kernel phase analysis.

\section{Data reduction}

At the time of our observations, the southern binary of the $\mathrm{T}$ Tau triple system was separated by $\sim 64$ mas, which is equivalent to $0.28,0.26,0.24$, and $0.20 \lambda / D$ in the ARIII, SIV_1, SIV_2, and NEII filters, respectively. Such small separations are very challenging for high-resolution single-dish imaging. Since VISIR-NEAR is mounted at the UT4, which also hosts the AOF, the instrument achieves extremely high Strehl ratios in the midinfrared. This makes our data set ideal for an analysis with the kernel phase technique, which models a single-dish telescope as an interferometer during the post-processing of the images. The kernel phase technique relies on a linear relationship between the pupil plane phase and the Fourier phase of the images, possible 

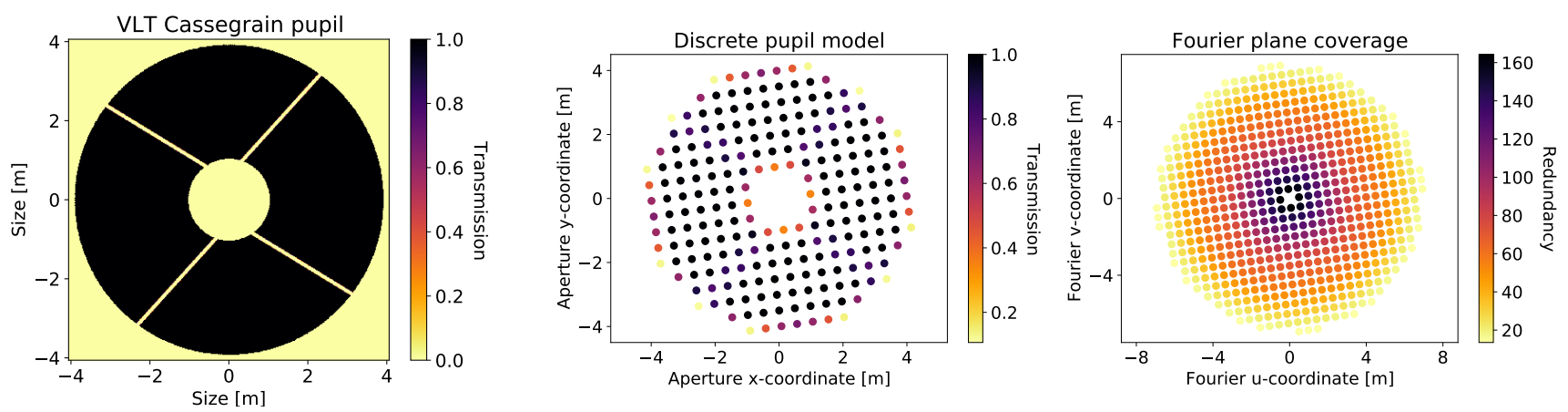

Fig. 1. VISIR-NEAR pupil model discretized onto a grid of subapertures for the kernel phase analysis. Left: VLT Cassegrain pupil with an extended central obscuration due to the M3 baffle and four thin M2 support struts ("spiders"), rotated by 81.8 deg clockwise. Middle: our discrete pupil model consisting of 224 subapertures, spanning 310 distinct baselines with 88 kernel phases. Right: Fourier plane coverage of our discrete pupil model.

only in the high-Strehl regime. With the known astrometry of the $\mathrm{T}$ Tau triple system from observations at shorter wavelengths, we can use the kernel phase technique to obtain its photometry in the mid-infrared.

\subsection{Kernel phase technique}

The kernel phase technique was developed by Martinache (2010), who was able to achieve an angular resolution of $\sim 0.6 \lambda / D$ on the known low-contrast binary GJ 164 with the Near-Infrared Camera and Multi-Object Spectrometer (NICMOS) on the Hubble Space Telescope (HST). Later, Pope et al. (2013) used the technique to detect brown dwarf companions, also with HST/NICMOS, and Pope et al. (2016) and Sallum \& Skemer (2019) showed that kernel phase should outperform sparse aperture masking under appropriate seeing conditions longward of $\sim 3 \mu \mathrm{m}$ from the ground given the reduced sensitivity to sky background noise. Recently, Kammerer et al. (2019) used the kernel phase technique to detect eight stellar companions in an archival VLT/Nasmyth Adaptive Optics System (NAOS) near-infrared imager and spectrograph (NACO) high-contrast imaging survey, two of which are below the classical diffraction limit at angular separations of $\sim 0.8$ and $\sim 1.2 \lambda / D$.

The kernel phase technique achieves its high resolution by making use of the superior calibration capabilities of the Fourier plane phase (i.e., the phase of the Fourier transform of the image) in the high-Strehl regime. Here, one can linearize the expression of the Fourier plane phase $\phi$ as a function of the pupil plane phase $\varphi$, that is,

$\phi(\varphi)=\boldsymbol{R}^{-1} \cdot \boldsymbol{A} \cdot \varphi+\phi_{\mathrm{obj}}+O\left(\varphi^{3}\right)$,

where $\boldsymbol{A}$ is the matrix of a linear map between a pair of pupil plane positions and their corresponding spatial frequency, $\boldsymbol{R}$ is a diagonal matrix encoding the redundancy of the spatial frequencies, and $\phi_{\mathrm{obj}}$ is the Fourier plane phase of the object intensity distribution (i.e., of the astronomical source). The first step was to model the single-dish telescope as an interferometer by discretizing the telescope pupil onto a grid of so-called subapertures in order to find a simple representation of matrix $\boldsymbol{A}$, and our VISIR-NEAR pupil model is shown in Fig. 1. In the high-Strehl regime, the higher order pupil plane phase noise $O\left(\varphi^{3}\right)$ is negligible and one can multiply Eq. (1) with the kernel $\boldsymbol{K}$ of $\boldsymbol{R}^{-1} \cdot \boldsymbol{A}$ to obtain the kernel phase $\theta$, namely,

$\theta=\boldsymbol{K} \cdot \phi=\underbrace{\boldsymbol{K} \cdot \boldsymbol{R}^{-1} \cdot \boldsymbol{A} \cdot \varphi}_{=0}+\boldsymbol{K} \cdot \phi_{\mathrm{obj}}+\mathcal{O}\left(\varphi^{3}\right) \approx \theta_{\mathrm{obj}}$.
This is a very powerful finding as it means that, to the second order, the kernel phase measured in the image is directly equivalent to the kernel phase of the astronomical source.

\subsection{Basic cleaning}

Table 1 shows an observing log for program 60.A-9107(E). Each chopping cycle consists of two chopping positions, at each of which NDIT exposures are saved and averaged into a single frame by the instrument control software. Then, for each of the $N_{\text {chop }}$ chopping cycles, we subtracted the two frames from the different chopping positions from each other in order to remove the mid-infrared background and averaged that over the $N_{\text {chop }}$ background-subtracted frames; doing so, we obtained one cleaned frame for each of the $N_{\text {avg }}$ observations. We note that we also nodded along the chopping axis during the observations but performed no nodding subtraction here for the sake of simplicity.

Next, we found the brightest PSF in the field-of-view (FOV) by looking for the brightest pixel in the median filtered (3 pixel) frame and cropped the frames to a size of $128 \times 128$ pixels around the brightest PSF, which corresponds to an FOV of $5.792 \operatorname{arcsec}^{2}$. A more sophisticated sub-pixel re-centering is performed at a later stage (see Sect. 3.3).

The detector position angle of each frame was computed as the arithmetic mean of the "POSANG" and "POSANG END" fits header keywords. This approximation is feasible since the observing times are short and the field rotation per fits file is only on the order of $0.25 \mathrm{deg}$. Unfortunately, the detector position angle computed from the fits header keywords does not represent the true position angle of the detector. There is an additional rotation offset $\vartheta$ that we determined from VISIR-NEAR data of the nearby and well-known binary system $\alpha$ Centauri. With the known astrometry of this binary system, we obtain a rotation offset of $\vartheta=94.02 \pm 0.10 \mathrm{deg}$ and a pixel scale of $45.25 \pm 0.10$ mas. The detector position angle and the rotation offset were both measured clockwise (i.e., west of north), while the position angle of the companions was measured counterclockwise (i.e., east of north), in accordance with the standard convention.

\subsection{Kernel phase extraction and calibration}

To extract the kernel phase from the cleaned frames, we used the XARA $^{1}$ package (Martinache 2010, 2013). XARA requires a discrete pupil model of the VISIR-NEAR instrument in order

1 https://github.com/fmartinache/xara/ 

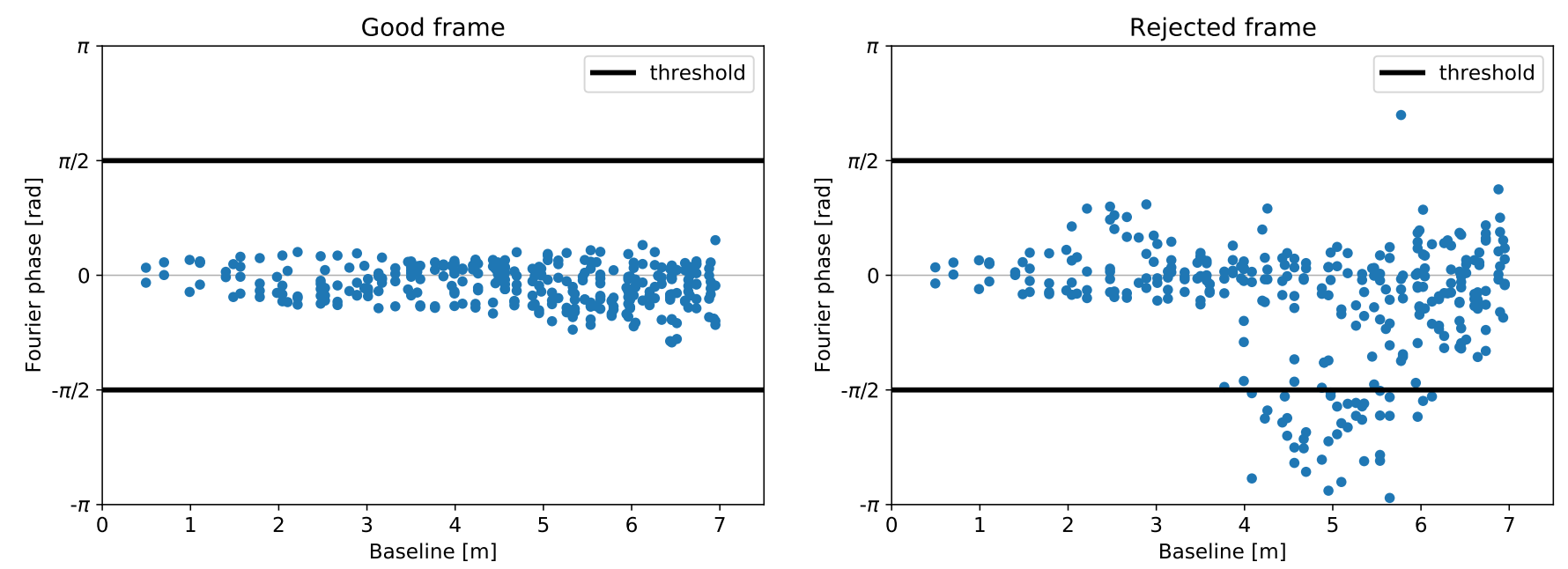

Fig. 2. Frame selection criterion used to reject bad frames that are not suitable for a kernel phase analysis. Left: Fourier phase $\phi$ as a function of baseline for an example frame taken with the SIV_1 filter that passes our selection criterion $(|\phi|<90 \mathrm{deg})$. Right: same, but for an example frame that does not pass our selection criterion due to seeing-driven aberrations.

to compute the kernel matrix $\boldsymbol{K}$. Figure 1 shows the VLT Cassegrain pupil (left-hand panel), our discrete pupil model consisting of 224 subapertures placed on a grid with a spacing of $0.5 \mathrm{~m}$ (middle panel), and the Fourier plane coverage of our discrete pupil model (right-hand panel). The use of subapertures with a continuous transmission between 0 and 1 (referred to as gray apertures) helps to significantly minimize residual calibration errors (Martinache et al. 2020). We note that the pupil is rotated by $81.8 \mathrm{deg}$ clockwise (west of north, see Fig. 1) with respect to the detector and we aligned the grid of subapertures with the pupil in order to obtain a symmetric pupil model and reduce calibration errors (see Martinache et al. 2020). We determined this rotation from VISIR-NEAR data of $\alpha$ Centauri, which feature bright diffraction artifacts from the secondary mirror (M2) support spiders.

XARA first masks the cleaned frames with an exponential windowing function $m$ (sometimes called super-Gaussian),

$m(x, y)=\exp \left(-\left(\frac{\sqrt{\left(x-x_{0}\right)^{2}+\left(y-y_{0}\right)^{2}}}{r}\right)^{4}\right)$,

with a radius $r$ of 50 pixels ( 2.263 arcsec) in order to minimize edge effects during the Fourier transform, where $x$ and $y$ are the $x$ - and $y$-coordinates of the frame and $x_{0}=64$ and $y_{0}=64$ are the coordinates of the center of the frame. This is equivalent to a Gaussian smoothing in the Fourier plane, and a value of 50 pixels is large enough to not interfere with the spatial frequencies measured by the VLT. It then extracts the Fourier plane phase $\phi$ from the cleaned (dither-subtracted) frames using a discrete Fourier transform at the uv-positions corresponding to the subapertures of our pupil model. Finally, it performs sub-pixel re-centering in the complex visibility space by multiplying the Fourier phase $\phi$ with a wedge function and computes the kernel phase $\theta=\boldsymbol{K} \cdot \phi$. This wedge function is determined by computing the photocenter of the brightest PSF on an iteratively shrinking window.

Furthermore, we estimated the kernel phase covariance $\boldsymbol{\Sigma}_{\theta}$ based on the pixel-to-pixel background variance in the cleaned frames. Therefore, we applied the basis transform

$\boldsymbol{\Sigma}_{\theta}=\boldsymbol{B} \cdot \boldsymbol{\Sigma}_{d} \cdot \boldsymbol{B}^{T}$,

where

$\boldsymbol{B}=\boldsymbol{K} \cdot \frac{\operatorname{Im}(\boldsymbol{F})}{|\boldsymbol{F} \cdot \boldsymbol{d} \cdot \boldsymbol{g} \cdot \boldsymbol{m}|}$ and $\boldsymbol{\Sigma}_{d}$ is the variance frame (see Kammerer et al. 2019). Here, we obtain the variance frame directly from the frame itself, namely,

$\Sigma_{d}=\operatorname{var}(\boldsymbol{d} \cdot g) \cdot \boldsymbol{m}^{2}$,

where $\boldsymbol{d}$ is the frame itself and $g$ is the gain in photoelectrons per detector count. We note that we masked out the center of the frame with a circular aperture of 80 pixels $(3.620$ arcsec) in diameter in order to avoid confusion from the PSF of T Tau.

Before continuing with the calibration and the model fitting, we made a frame selection based on the absolute Fourier phase $|\phi|$. We rejected any frame whose absolute Fourier phase $|\phi|$ exceeds $90 \mathrm{deg}$ on at least one of the 310 baselines, that is to say, vectors connecting two subapertures (see Fig. 2). This threshold is chosen empirically and to be sufficiently high so that frames with a high-Strehl PSF of T Tau are kept (we note that T Tau is a low-contrast triple system and therefore has a nonzero Fourier phase) but that frames suffering seeing-driven aberrations are rejected. For frames whose absolute Fourier phase $|\phi|$ exceeds $90 \mathrm{deg}$, higher order pupil plane phase errors are large and would add significant systematic errors. The number of good frames $N_{\text {good }}$ obtained for T Tau and the PSF reference for each filter can be found in Table 1 . The number of rejected frames can be obtained as the difference between $N_{\text {avg }}$ and $N_{\text {good }}$.

In order to calibrate the kernel phase, we subtracted the average of the kernel phase measured on the calibrator from the kernel phase measured on the science target (see e.g., Martinache 2010). The corresponding kernel phase covariance then writes as

$\boldsymbol{\Sigma}_{\theta}=\boldsymbol{\Sigma}_{\theta, \mathrm{sci}}+\frac{1}{N_{\text {good }}^{2}} \sum_{n=1}^{N_{\text {good }}} \boldsymbol{\Sigma}_{\theta, \mathrm{cal}, n}$

\subsection{Model fitting}

With the kernel phase $\theta$ and its covariance $\boldsymbol{\Sigma}_{\theta}$, we can perform model fitting based on likelihood maximization or $\chi^{2}$ minimization. For this, we used a model of $N$ point sources,

$\theta_{\mathrm{mod}}=\boldsymbol{K} \cdot \arg \left(\sum_{n=1}^{N} \xi_{n} c_{n} \exp \left(-2 \pi i\left(\frac{\Delta_{\mathrm{RA}, n} u}{\lambda}+\frac{\Delta_{\mathrm{Dec}, n} v}{\lambda}\right)\right)\right)$,

A36, page 4 of 12 
where $\xi_{n}$ is the relative flux of the $n$th component (normalized to the total flux), $c_{n}$ is the complex visibility of the $n$th component, $\Delta_{\mathrm{RA}, n}$ and $\Delta_{\mathrm{Dec}, n}$ are the sky offset of the $n$th component from the center of the frame, $u$ and $v$ are the Fourier $u$ - and $v$-coordinates of our pupil model, and $\lambda$ is the observing wavelength. In case of unresolved point sources (such as is the case for our observations), the complex visibility $c_{n}=1$.

The $N$-band flux of both T Tau Sa and $\mathrm{Sb}$ is entirely dominated by emission from their CSDs. Both CSDs are tidally truncated by the binary's orbit, which has a semi-major axis of $\sim 12.5$ au and an eccentricity of $\sim 0.55$ (Schaefer et al. 2020). For such orbital parameters, Artymowicz \& Lubow (1994) predict a ratio of the disk truncation radius to the semimajor axes that would be on the order of $\sim 0.25$ (=3.1 au) for T Tau Sa and $\sim 0.15$ $(=1.9 \mathrm{au})$ for $\mathrm{Sb}$. This corresponds to angular sizes of 21 mas and 13 mas, respectively, for the CSDs of T Tau Sa and Sb and is significantly smaller than the diffraction limit at our shortest observing wavelength of $\sim 230$ mas. In addition, the CSDs get warmer and brighter toward the inner disk rim, with radii of a few tenths of an au ( $\lesssim 3$ mas). Therefore, we can safely assume that $\mathrm{T}$ Tau $\mathrm{Sa}$ and $\mathrm{Sb}$ are spatially unresolved in our data.

In the following, we used a more intuitive representation $f_{n}$ of the relative flux of the individual components by normalizing it to the flux of the brightest component so that $f_{1}=1$ and $f_{n>1} \leq$ 1 . The $\xi_{n}$ are related to the $f_{n}$ according to

$\xi_{n}=\frac{f_{n}}{\sum_{n=1}^{N} f_{n}}$.

If we are in the high-contrast regime $\left(\xi_{n>1} \ll 0.5\right)$ and only fit for two components, the model can be simplified by linearization (Kammerer et al. 2019) and by putting the brighter component into the center of the frame $\left(\Delta_{\mathrm{RA}, 1}=\Delta_{\mathrm{Dec}, 1}=0\right)$. Then, we can solve analytically for the relative companion flux,

$\xi_{2}=\frac{\theta_{\text {ref }}^{T} \cdot \Sigma_{\theta}^{-1} \cdot \theta}{\theta_{\text {ref }}^{T} \cdot \Sigma_{\theta}^{-1} \cdot \theta_{\text {ref }}}$,

on an RA-Dec grid, where $\theta_{\text {ref }}$ is the binary model (Eq. (8) for $N=2$ ) evaluated on an RA-Dec grid and for a small relative reference flux $\xi_{2 \text {,ref }} \ll 0.5$ (see Kammerer et al. 2019). We note that with the T Tau system, we are not in the high-contrast regime where this simplification holds. Nevertheless, such a grid search allows us to find a good first guess for the relative companion flux and its position, which we can then use as an initial position for a least-squares or a Markov chain Monte Carlo (MCMC) algorithm. Such algorithms directly minimize the $\chi^{2}$ of the model, that is,

$\chi^{2}=R^{T} \cdot \Sigma_{\theta}^{-1} \cdot R$,

where $R=\theta-\theta_{\text {mod }}$ is the residual kernel phase signal between the data and the model, so that no linearization is necessary.

\section{Results and analysis}

Our goal is to determine the flux of $\mathrm{T} \mathrm{Tau} \mathrm{Sa} \mathrm{and} \mathrm{Sb}$ relative to $\mathrm{T}$ Tau $\mathrm{N}$ in all four filters. Therefore, we first performed a binary model fit in order to find the combined flux of T Tau $\mathrm{S}$ (i.e., $\mathrm{T}$ Tau $\mathrm{Sa}$ and $\mathrm{Sb}$ ) relative to $\mathrm{T}$ Tau $\mathrm{N}$. Then, we performed a triple model fit using half of the combined relative flux of $\mathrm{T}$ Tau $\mathrm{S}$ as initial values for the fluxes of $\mathrm{T}$ Tau $\mathrm{Sa}$ and $\mathrm{Sb}$ relative to $\mathrm{T}$ Tau $\mathrm{N}$ (making the simple assumption that $\mathrm{T}$ Tau $\mathrm{Sa}$ and $\mathrm{Sb}$ are equally bright; the least-squares or MCMC algorithm will then find the true flux ratio). We note that for the NEII filter we proceeded slightly differently since $\mathrm{T}$ Tau $\mathrm{S}$, and not $\mathrm{T}$ Tau $\mathrm{N}$, is the brightest component (see Sect. 4.3). Finally, we created mock data with a range of different fluxes for $\mathrm{T}$ Tau $\mathrm{Sa}$ and $\mathrm{Sb}$ relative to $\mathrm{T}$ Tau $\mathrm{N}$ and repeated the triple model fits in order to demonstrate that our kernel phase model fitting technique correctly reproduces the mock data.

\subsection{Binary model fits}

Figure 3 shows the results of our binary model fits to the $\mathrm{T}$ Tau system, with both position and flux left as free parameters, yielding a combined flux of $\mathrm{T}$ Tau $\mathrm{S}$ relative to T Tau N of $0.546 \pm 0.015$ (ARIII), $0.270 \pm 0.064$ (SIV_1), $0.464 \pm 0.051$ (SIV_2), and 1.217 \pm 0.047 (NEII). The position uncertainties are on the order of $\sim 10$ mas, constrained by the astrometric precision of the kernel phase technique; the best fit position varies slightly from filter to filter, also due to the changing flux ratio between $\mathrm{T}$ Tau Sa and $\mathrm{Sb}$, which shifts the photocenter of the combined southern component. We note that the NEII data are centered on T Tau S, which is the brighter component at $12.80 \mu \mathrm{m}$. This already reveals some differences compared to the values reported by Skemer et al. (2008), who found the combined southern component to be brighter than the northern component at $8.7 \mu \mathrm{m}$ ( $\sim$ ARIII). At $10.55 \mu \mathrm{m}\left(\sim \mathrm{SIV} \_2\right)$, our result of a flux ratio of $0.464 \pm 0.051$ between the southern and the northern component is more similar to the value of $\sim 0.57$ reported by Skemer et al. (2008), and we find the combined southern component to only be brighter than the northern component at the longest wavelength $(12.80 \mu \mathrm{m})$.

Since $\mathrm{T}$ Tau $\mathrm{N}$ is fainter than $\mathrm{T}$ Tau $\mathrm{S}$ at $12.80 \mu \mathrm{m}$ (NEII), the kernel phase analysis is slightly more complicated. Therefore, we only report on the analysis of the ARIII, SIV_1, and SIV_2 data in the following and treat the NEII data separately in Sect. 4.3. Furthermore, the reduced $\chi^{2}$ of our binary model fits is larger than one, meaning that we are underestimating the errors. This is in agreement with previous studies that have shown that the kernel phase technique is dominated by systematic errors, usually yielding a reduced $\chi^{2}$ between $\sim 1-10$ (Martinache et al. 2020; Laugier et al. 2020; Kammerer et al. 2019; Ireland 2013).

\subsection{Triple model fits}

For the triple model fits, we used the known offsets of $\mathrm{T}$ Tau $\mathrm{Sa}$ and $\mathrm{Sb}$ relative to $\mathrm{T}$ Tau $\mathrm{N}$, which are $(-648.1$ mas $\mathrm{N}$, -188.5 mas E) for T Tau Sa and (-595.6 mas N, -151.4 mas E) for $\mathrm{T}$ Tau Sb. We obtained these values from extrapolating the orbits of the T Tau system from Köhler et al. (2016), which were obtained from VLT/SPHERE astrometry, for the date of our observations. Their accuracy is $\sim 1$ mas.

Figure 4 shows the results of our triple model fits to the T Tau system, with only the fluxes of T Tau $\mathrm{Sa}$ and $\mathrm{Sb}$ relative to T Tau $\mathrm{N}$ left as free parameters. We did both a simple minimization based on a Broyden-Fletcher-Goldfarb-Shanno least-squares algorithm (left-hand panels) and an MCMC fit using emcee ${ }^{2}$ (Foreman-Mackey et al. 2013, right-hand panels). In both cases, we used half of the combined relative flux of T Tau $\mathrm{S}$ as initial values for the fluxes of $\mathrm{T}$ Tau $\mathrm{Sa}$ and $\mathrm{Sb}$ relative to $\mathrm{T}$ Tau $\mathrm{N}$. Moreover, we restricted the search space to $0 \leq f_{2,3} \leq 1$ and normalized the log-likelihood function minimized with the MCMC algorithm by a temperature equal to the reduced $\chi^{2}$ of the best fit triple model (see the left-hand panels of Fig. 4) in order to artificially inflate the uncertainties. As can be seen in the corner plots, there is a strong anti-correlation between the relative fluxes

\footnotetext{
2 https://github.com/dfm/emcee
} 

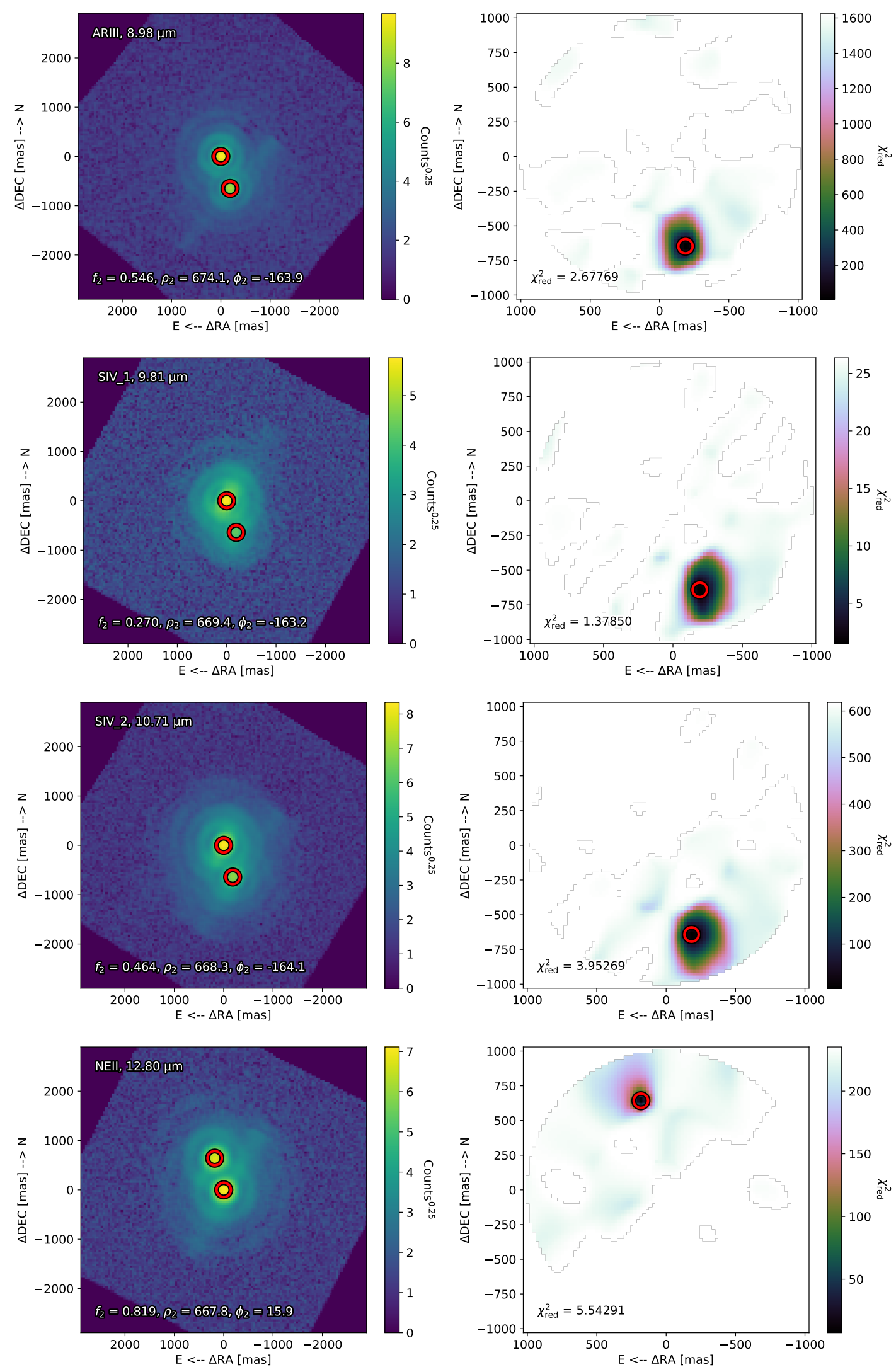

Fig. 3. De-rotated and co-added VISIR-NEAR images of the T Tau triple system and the two-component fit (left-hand column, red circles) and the corresponding maps of the reduced $\chi^{2}$ (right-hand column) in the ARIII, SIV_1, SIV_2, and NEII filters (from top to bottom). In the right-hand panels, the red circle highlights the best fit position for the companion (here, the companion is assumed to be the combined PSF of T Tau Sa and $\mathrm{Sb}$ ). The relative flux $f_{2}$, the angular separation $\rho_{2}$ [mas], and the position angle $\phi_{2}$ [deg] of the best fit are printed in the left-hand panels, and its reduced $\chi^{2}$ is printed in the right-hand panels. We note that the NEII data are centered on T Tau $\mathrm{S}$, which is the brighter component at $12.80 \mu \mathrm{m}$. 

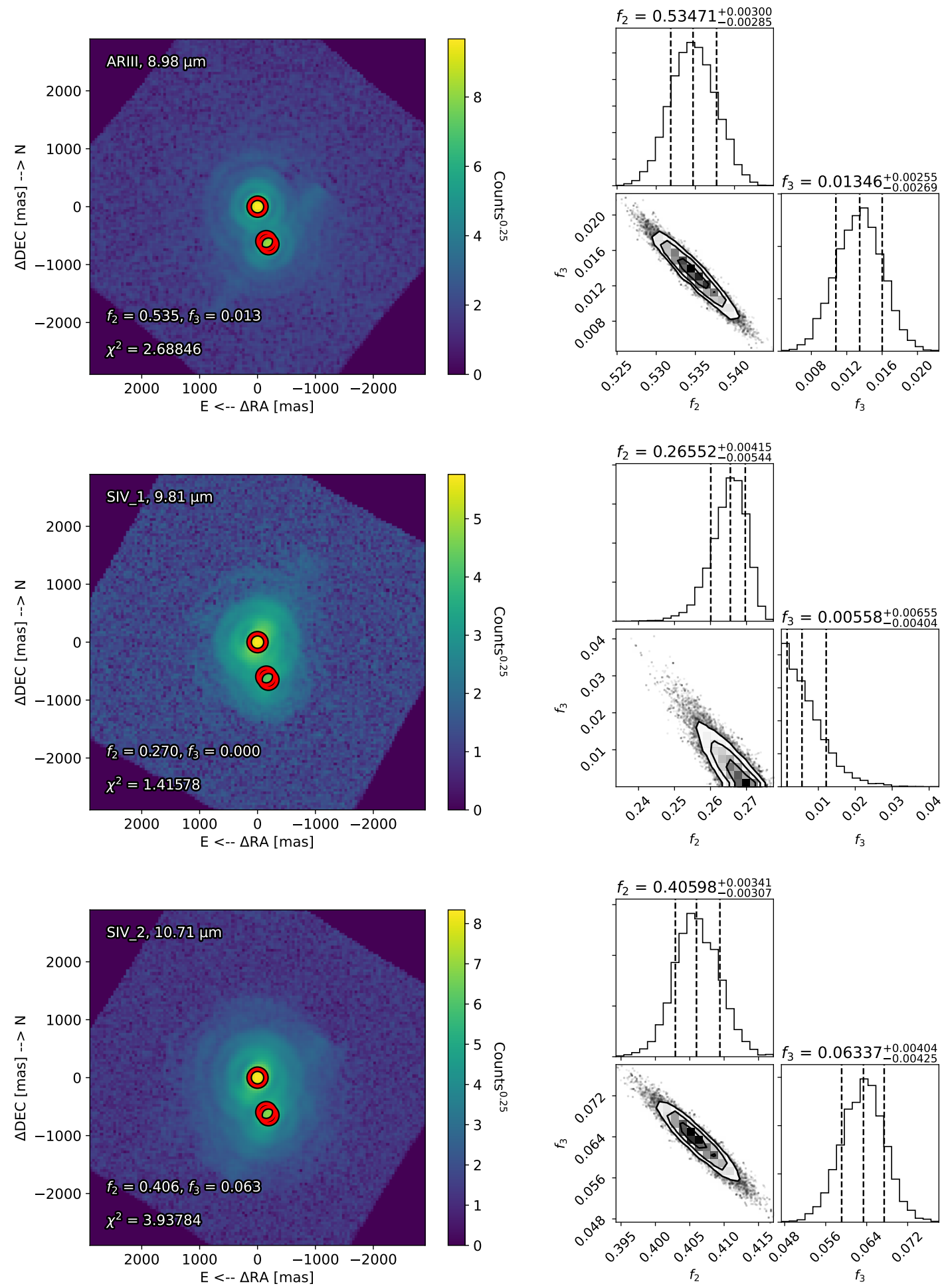

Fig. 4. De-rotated and co-added VISIR-NEAR images of the T Tau triple system and the three-component fit (left-hand column, red circles) and the corresponding MCMC corner plots (Foreman-Mackey 2016, right-hand column) in the ARIII, SIV_1, and SIV_2 filters (from top to bottom).

of T Tau Sa and Sb caused by the fact that their combined flux is constrained by the data. Furthermore, $\mathrm{T}$ Tau $\mathrm{Sb}$ is fainter than $\mathrm{T}$ Tau Sa in all three filters, which is similar to the results from Skemer et al. (2008), and it is consistent with providing zero flux at $9.81 \mu \mathrm{m}$.

Since we estimated the accuracy of the fixed VISIR-NEAR position angle offset $\vartheta$ determined from $\alpha$ Centauri data to be $\sim 0.10 \mathrm{deg}$, we repeated the MCMC fit with $\vartheta$ and the fluxes of
$\mathrm{T}$ Tau Sa and Sb relative to T Tau N as free parameters, simultaneously for the ARIII, SIV_1, and SIV_2 data (see Fig. 5). This approach allows us to explore the entire parameter space and to assess the errors originating from the uncertainty in the position angle offset $\vartheta$. The results are similar to when the position angle offset $\vartheta$ is fixed and each filter is fitted separately, with similar uncertainties, suggesting that the uncertainty in the position angle offset $\vartheta$ does not significantly limit our precision. We note 


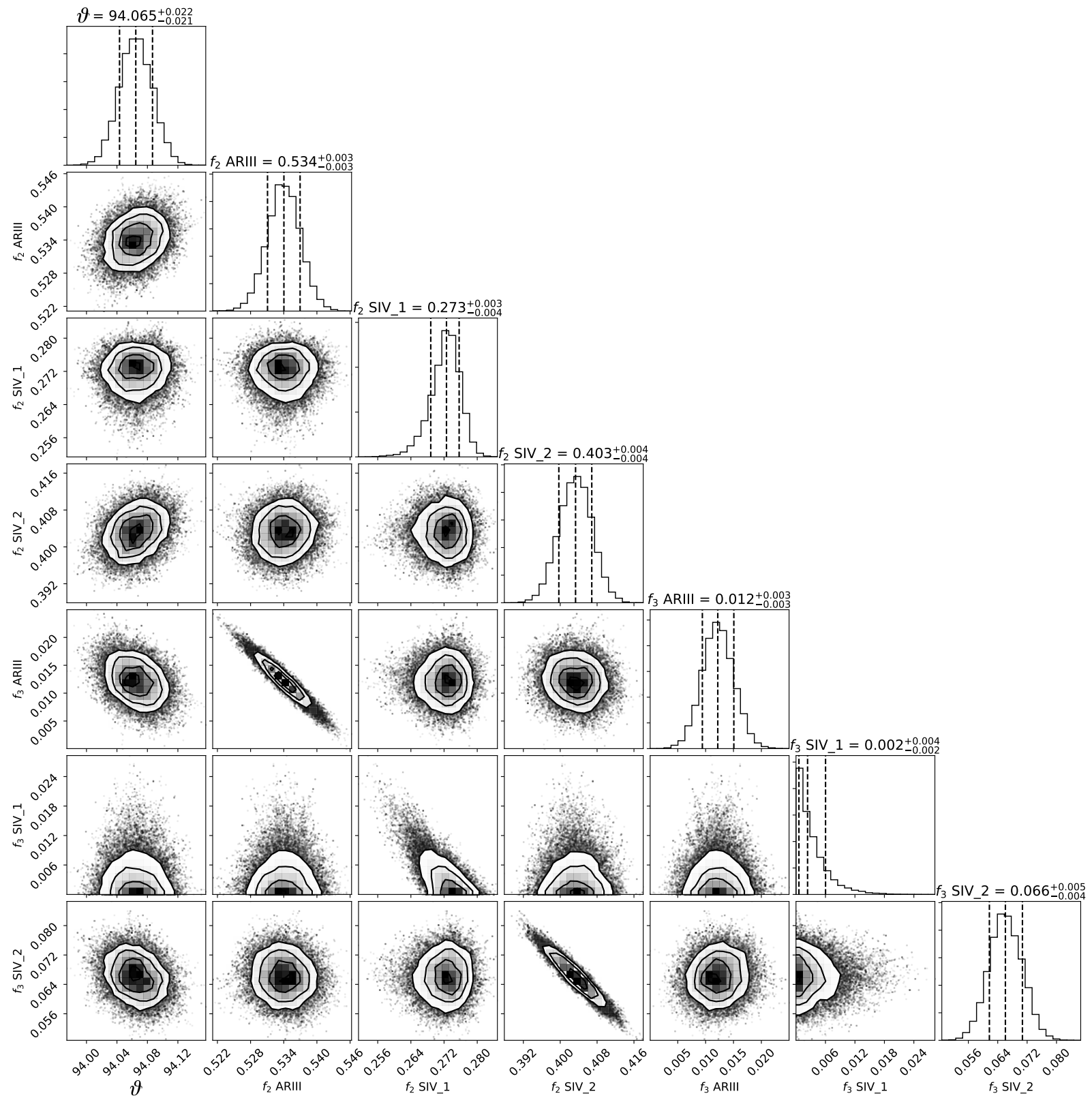

Fig. 5. Corner plot (Foreman-Mackey 2016) of an MCMC fit of the kernel phase triple model with the rotation offset $\phi$ and the relative fluxes of T Tau $\mathrm{Sa}\left(f_{2}\right)$ and $\mathrm{Sb}\left(f_{3}\right)$ as free parameters, simultaneously for the ARIII, SIV_1, and SIV_2 data.

that the best fit position angle offset $\vartheta_{\text {fit }}=94.07 \pm 0.02$ is consistent with our assumption of $\vartheta_{\text {prior }}=94.02 \pm 0.10$ that is based on $\alpha$ Centauri data.

Finally, we repeated the simultaneous triple model fits while varying the position offsets of $\mathrm{T}$ Tau $\mathrm{Sa}$ and $\mathrm{Sb}$ by \pm 1 mas in order to empirically estimate the errors originating from the $\sim 1$ mas uncertainty in these offsets. Again, we find similar results within the uncertainties, suggesting that the uncertainty in the position offsets of $\mathrm{T} \mathrm{Tau} \mathrm{Sa} \mathrm{and} \mathrm{Sb} \mathrm{does} \mathrm{not} \mathrm{significantly} \mathrm{limit}$ our precision either.

\subsection{NEIl data}

In the NEII data, at $12.80 \mu \mathrm{m}$, the southern binary of the $\mathrm{T}$ Tau triple system is separated by only $0.2 \lambda / D$. Moreover, it is brighter than $\mathrm{T}$ Tau N, so we had to center the frames on T Tau $\mathrm{S}$ before extracting the kernel phase (only if the brightest PSF is in the center of the frames can we avoid the Fourier phase $\phi$ wrapping around $\pm \pi$ and leading to discontinuities and a corrupt kernel phase $\theta$ ).

In order to perfectly center the frames on $\mathrm{T}$ Tau Sa, we first used XARA's sub-pixel re-centering routine to center the frames on $\mathrm{T}$ Tau $\mathrm{N}$. We then computed the exact shift that we have to apply to the frames in order to put $\mathrm{T}$ Tau $\mathrm{Sa}$ into the center. This is possible because we know its $\left(\Delta_{\mathrm{RA}}, \Delta_{\text {Dec }}\right)$ offset from VLT/SPHERE astrometry. Directly centering on $\mathrm{T}$ Tau $\mathrm{Sa}$ is impossible since its PSF is unresolved with the PSF of T Tau Sb; as such, XARA's re-centering routine would only find the combined photometric center of $\mathrm{T}$ Tau $\mathrm{Sa}$ and $\mathrm{Sb}$. Then, we proceeded similarly to how we treated the ARIII, SIV_1, and SIV_2 data and fit the kernel phase binary and triple models to the data. However, for the triple model fits, the offsets of $\mathrm{T}$ Tau $\mathrm{N}$ and $\mathrm{Sb}$ relative to the center of the frames (i.e., T Tau $\mathrm{Sa}$ ) are now (648.1 mas N, 188.5 mas E) and (52.5 mas N, 37.1 mas E). 

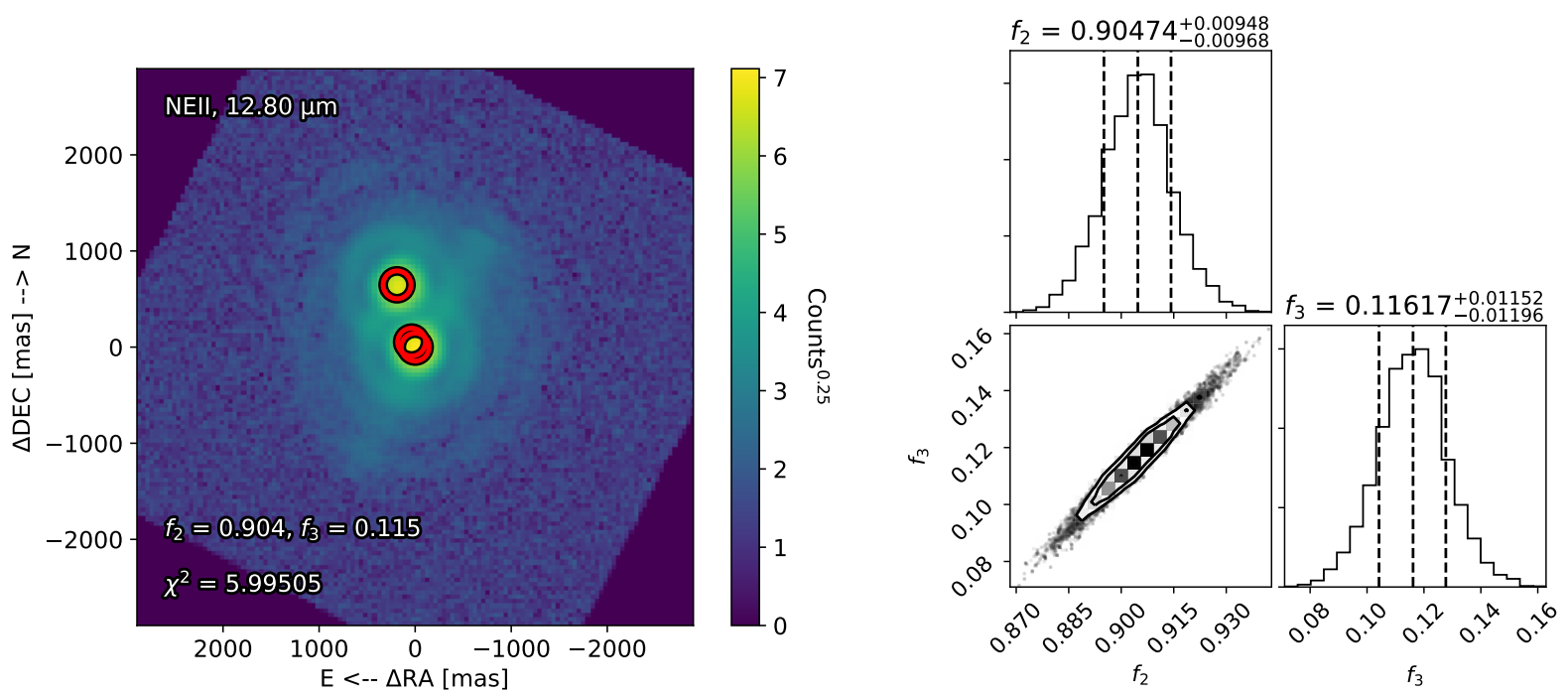

Fig. 6. Same as Fig. 4 but for the NEII filter and with the relative fluxes of $\mathrm{T}$ Tauri $\mathrm{N}\left(f_{2}\right)$ and $\mathrm{Sb}\left(f_{3}\right)$ as free parameters.

Our triple model fit results are shown in Fig. 6. We note that there is now a positive correlation between the relative fluxes of $\mathrm{T}$ Tau $\mathrm{N}$ and $\mathrm{Sb}$ since they are both measured relative to the same PSF of T Tau Sa and are well resolved from each other.

\subsection{Mock data}

In order to validate our approach, we created mock data of the T Tau system and tried to recover it using our kernel phase model fitting techniques. Therefore, we used the calibrator HD 27639 as a reference PSF. Then, we shifted, normalized, and co-added this reference PSF so that it models the three components of the $\mathrm{T}$ Tau system. For each filter, we modeled one system with the observed flux ratios and three more systems with $\mathrm{T}$ Tau $\mathrm{Sa} / \mathrm{Sb}$ flux ratios of $0.5 / 0.5,0.75 / 0.25$, and $0.9 / 0.1$ (T Tau $\mathrm{Sa} / \mathrm{Sb}$ flux ratios of $1 / 0.5,1 / 0.25$, and $1 / 0.1$ for the NEII filter). In all scenarios, the combined flux of $\mathrm{T}$ Tau $\mathrm{Sa}$ and $\mathrm{Sb}$ relative to $\mathrm{T}$ Tau $\mathrm{N}$ (the flux of T Tau N for the NEII filter) is equal to the value that we obtained from the binary model fit (see Fig. 3). We applied the kernel phase technique to the mock data in exactly the same fashion as we did to the real data (e.g., a different re-centering method for the ARIII, SIV_1, and SIV_2 data compared to the NEII data).

Table A.1 reports the simulated and recovered relative fluxes for all scenarios and the ARIII, SIV_1, SIV_2, and NEII filters. The initial values for $f_{2}$ and $f_{3}$ were always set to half of the combined flux of T Tau S relative to T Tau N. We note that for the NEII filter, the relative flux of $\mathrm{T}$ Tau $\mathrm{Sa}$ is fixed to one and we are fitting for the relative fluxes of $\mathrm{T}$ Tau $\mathrm{N}$ and $\mathrm{Sb}$.

In most cases, the recovered relative fluxes are consistent with the simulated ones within two sigma, and in all cases within three sigma. Given that the fits with mock data do not account for systematic errors and that our covariance model underestimates the uncertainties (see Sect. 4.1), differences of two to three sigma are expected. This leads us to the conclusion that the photometry obtained from our kernel phase model fitting techniques is reliable down to angular separations of $\sim 0.2 \lambda / D$ (that is the angular separation of $\mathrm{T} \mathrm{Tau} \mathrm{Sa} \mathrm{and} \mathrm{Sb}$ at the longest wavelength in the NEII filter). However, it is also clear that our techniques are limited by systematic errors, and not the uncertainty in the fixed position angle offset $\vartheta$ or the $\left(\Delta_{\mathrm{RA}}, \Delta_{\text {Dec }}\right)$ offset of T Tau $\mathrm{Sa}$ and $\mathrm{Sb}$ relative to $\mathrm{T}$ Tau $\mathrm{N}$ obtained from VLT/SPHERE astrometry. This can be concluded from the relatively large discrepancies between the simulated and recovered fluxes when compared to the uncertainties predicted by the MCMC fit, which accounts for the largest statistical noise (photon noise). Hence, we empirically estimated the uncertainties of the relative fluxes from the observed differences between the simulated and recovered values for the mock data (see Table 2). We adapted a conservative uncertainty of $\sigma_{\mathrm{emp}}=0.02$ for the ARIII, SIV_1, and SIV_2 filters, given that all observed differences fall within this range, and a slightly larger uncertainty of $\sigma_{\text {emp }}=0.03$ for the NEII filter, given the slightly larger observed differences due to the longer wavelength and the different data reduction approach.

\subsection{Photometry}

The final photometry of T Tau N, Sa, and Sb was computed from the kernel phase relative fluxes and the PSF photometry with an aperture of 70 pixels in diameter on the cleaned frames of T Tau and HD 27639 (the calibrator). HD 27639 is part of the VISIR ${ }^{3}$ photometric standards list, from which we adapted fluxes of $14.9 \pm 0.3 \mathrm{Jy}(\mathrm{ARIII}), 13.1 \pm 0.3 \mathrm{Jy}\left(\mathrm{SIV}_{-} 1\right), 11.3 \pm 0.3 \mathrm{Jy}$ (SIV_2), and $8.7 \pm 0.3 \mathrm{Jy}$ (NEII) with conservative uncertainties. Our final photometry for the T Tau system, using the results from Figs. 4 and 6 for the relative fluxes, is reported in Table 2 and shown in Fig. 7.

\section{Discussion}

Figure 7 shows the photometry of T Tau N, Sa, and Sb in the mid-infrared over a period of $\sim 15$ years. All three components were resolved with VLTI/MIDI interferometry in November 2004 (Ratzka et al. 2009), with the MMT Mid-IR Array Camera 4 (MIRAC4) using high-contrast imaging in November 2006 (Skemer et al. 2008), and VLT/VISIR-NEAR kernel phase interferometry (this work) in December 2019.

The SED of $\mathrm{T}$ Tau $\mathrm{N}$ is quite stable over time, within the uncertainties, and confirms the consistent photometry typically obtained from this star. We also confirm the detection of weak silicate emission (Ghez et al. 1991), which suggests the presence of a face-on CSD around T Tau N. Instead, the SED of T Tau Sa

\footnotetext{
3 http://www . eso.org/sci/facilities/paranal/ instruments/visir/tools/zerop_cohen_Jy.txt
} 
Table 2. Best fit relative fluxes of the T Tau system from an MCMC fit of the kernel phase triple model to the VISIR-NEAR data.

\begin{tabular}{lccccc}
\hline \hline Filter & $\lambda[\mu \mathrm{m}]$ & Star & Rel. flux & $\sigma_{\text {emp }}$ & Flux [Jy] \\
\hline ARIII & 8.98 & $\mathrm{~N}$ & 1.000 & 0 & $8.12 \pm 0.13$ \\
ARIII & 8.98 & $\mathrm{Sa}$ & $0.535_{-0.003}^{+0.003}$ & 0.02 & $4.34 \pm 0.11$ \\
ARIII & 8.98 & $\mathrm{Sb}$ & $0.013_{-0.003}^{+0.003}$ & 0.02 & $0.10 \pm 0.08$ \\
\hline SIV_1 & 9.81 & $\mathrm{~N}$ & 1.000 & 0 & $9.81 \pm 0.16$ \\
SIV_1 & 9.81 & $\mathrm{Sa}$ & $0.266_{-0.005}^{+0.004}$ & 0.02 & $2.68 \pm 0.07$ \\
SIV_1 & 9.81 & $\mathrm{Sb}$ & $0.006_{-0.004}^{+0.007}$ & 0.02 & $0.02 \pm 0.05$ \\
\hline SIV_2 & 10.71 & $\mathrm{~N}$ & 1.000 & 0 & $10.04 \pm 0.19$ \\
SIV_2 & 10.71 & $\mathrm{Sa}$ & $0.406_{-0.003}^{+0.003}$ & 0.02 & $4.05 \pm 0.13$ \\
SIV_2 & 10.71 & $\mathrm{Sb}$ & $0.063_{-0.004}^{+0.004}$ & 0.02 & $0.66 \pm 0.10$ \\
\hline NEII & 12.80 & $\mathrm{~N}$ & $0.905_{-0.010}^{+0.009}$ & 0.03 & $9.63 \pm 0.43$ \\
NEII & 12.80 & $\mathrm{Sa}$ & 1.000 & 0 & $10.64 \pm 0.42$ \\
NEII & 12.80 & $\mathrm{Sb}$ & $0.116_{-0.012}^{+0.012}$ & 0.03 & $1.23 \pm 0.32$ \\
\hline
\end{tabular}

Notes. We also report an empirical uncertainty $\left(\sigma_{\text {emp }}\right)$ for the relative fluxes (see Sect. 4.4) as well as the final photometry of T Tau N, Sa, and $\mathrm{Sb}$ in all filters.

varies by up to a factor of four over the $N$-band, with our measurement falling approximately in between the values reported by Skemer et al. (2008) and Ratzka et al. (2009) at the shorter wavelengths. However, our $12.8 \mu \mathrm{m}$ flux falls significantly below the $N$-band fluxes measured in October $1990(17.1 \pm 2.3 \mathrm{Jy})$ and February 2008 (12.1-16.7 Jy) by Ghez et al. (1991) and van Boekel et al. (2010), respectively. So, while T Tau Sa is currently rather bright in the near-infrared (Schaefer et al. 2020) when compared to the 2004-2006 period, its $N$-band brightness is average at best. This is the well-known "bluer when brighter" behavior typical for extinction, but it is also consistent with variable accretion from a small edge-on CSD around $\mathrm{T}$ Tau Sa (van Boekel et al. 2010). Variability in the $\sim 9.7 \mu \mathrm{m}$ silicate feature has also been observed for other strongly accreting CTTSs, such as CW Tau, DG Tau, and XZ Tau (Leisenring et al. 2007).

For $\mathrm{T}$ Tau Sa, we also detect the silicate absorption feature known to exist in the $10 \mu \mathrm{m}$ spectral region around the southern component (Ghez et al. 1991). This feature was later shown to arise from extinction toward $\mathrm{T}$ Tau $\mathrm{Sa}$ and $\mathrm{Sb}$, but with a greater optical depth toward Sa (Ratzka et al. 2009). As pointed out by these authors, this difference in extinction must have been rather local given the small projected separation between $\mathrm{T}$ Tau Sa and $\mathrm{Sb}$ of $\sim 0.1$ arcsec or $\sim 15 \mathrm{au}$, and they attributed it to silicate absorption caused by the outer parts of T Tau Sa's CSD. However, van Boekel et al. (2010) showed that Sa's tidally truncated small disk should be warm enough even in its outer parts to produce silicate emission, including when seen edge-on. Therefore, additional extinction caused by, for example, the southern CBD is necessary to explain the SED of T Tau Sa.

In contrast to $\mathrm{T}$ Tau $\mathrm{Sa}$, our data show a significant dimming of T Tau Sb when compared to the 2004-2006 period. The effect is most prominent around the silicate feature, where $\mathrm{Sb}$ has been dimming by at least a factor of five or $\sim 2$ mag (see Fig. 7). This is consistent with the current $K$-band dimming of $\sim 2-$ $2.5 \mathrm{mag}$ observed over the same period (Schaefer et al. 2020) and enables us to infer an upper limit on the dust size by considering standard dust models (see e.g., Kruegel \& Siebenmorgen 1994) with a size distribution of $n(a) \sim a^{-3.5}$ (Mathis et al. 1977), which predict a bell-shaped silicate feature with a maximum near $9.7 \mu \mathrm{m}$. For these, the larger the maximum grain size to cut the power-law distribution is set, the wider and shallower the silicate feature becomes. For a maximum grain size well below $1 \mu \mathrm{m}$, which is typical for interstellar dust not processed in the dense inner regions of CSDs, the dust opacity around $10 \mu \mathrm{m}$ would be similar to that in the $K$-band, and therefore the extinction would also be similar.

Köhler \& Kubiak (2020) proposed that T Tau Sb has now moved along its orbit around Sa through the southern CBD and consequently suffers increased dust extinction. The Sa$\mathrm{Sb} \mathrm{CBD}$ is aligned roughly north to south, with an inclination of $\sim 60 \mathrm{deg}$ and a position angle of $\sim 30 \mathrm{deg}$ (see Fig. 1 of Köhler \& Kubiak 2020). Therefore, T Tau Sb was located behind the dense inner region of the Sa-Sb CBD, which extents out to $\sim 150$ mas (Yang et al. 2018) from the center of mass ( $\sim$ T Tau $\mathrm{Sa}$ ), at the time of our observations. Our photometry thus serves as evidence for increased dust extinction toward T Tau Sb caused by its orbital motion through the southern CBD and confirms the scenario proposed by Köhler \& Kubiak (2020). Another possible explanation would be a cloud of dust entering the observing beam in front of T Tau Sb. Such a clumpy structure would be motivated by the strong outflows and turbulence observed in the T Tau system (e.g., Kasper et al. 2016). However, since T Tau Sb was already observed to be faint by Koresko (2000) in data from 1997 (when it was on the opposite side of T Tau Sa; see Fig. 1 of Köhler \& Kubiak 2020), it seems more likely that the Sa-Sb CBD is responsible for its dimming.

Comparing our mid-infrared photometry from December 2019 to that from November 2006 and 2004, we see the silicate absorption variability of $\mathrm{T}$ Tau $\mathrm{Sb}$ that was introduced by its passage through the southern $\mathrm{CBD}$, but we do not claim variability of T Tau Sa's silicate feature. We note that van Boekel et al. (2010) measured variability at $12.8 \mu \mathrm{m}$ (i.e., not in the silicate feature) of the unresolved T Tau S binary over just a few days and attributed this to variable accretion, where UV emission is reprocessed by the disk and shows up in the mid-infrared. These authors also modeled T Tau Sa's truncated CSD and found that they cannot reproduce silicate absorption by the CSD alone (instead, Sa's small and warm CSD always shows silicate in emission) and that foreground extinction by cold dust (e.g., by the $\mathrm{CBD}$ ) is therefore needed.

\section{Conclusions}

In this paper, we measure the brightness of all three components of the iconic T Tau system in the mid-infrared, around $\sim 10 \mu \mathrm{m}$, in order to perform photometry and study the variability caused by its dynamic and dusty environment. We confirm weak silicate emission around $\mathrm{T}$ Tau $\mathrm{N}$, indicating a face-on CSD, and strong silicate absorption around $\mathrm{T}$ Tau Sa, consistent with extinction by southern circumbinary material (e.g., van Boekel et al. 2010). For T Tau Sb, we observe a dimming over the entire $\sim 8-13 \mu \mathrm{m}$ spectral range when compared to data from 2004 and 2006, which is in agreement with recent dimming in the near-infrared (K-band) observed by Schaefer et al. (2020).

The dimming of $\mathrm{T}$ Tau $\mathrm{Sb}$ observed over a wide spectral range (near- to mid-infrared) is consistent with $\mathrm{Sb}$ having moved behind the dense inner region of the Sa-Sb CBD, resulting in increased dust extinction. Therefore, we can confirm the scenario proposed by Köhler \& Kubiak (2020) and the Sa-Sb CBD geometry derived by Yang et al. (2018), which, together with previous works (e.g., Duchêne et al. 2002; Skemer et al. 2008; Ratzka et al. 2009), strengthen the evidence for a significant misalignment between the CBD and the T Tau Sa CSD. Most surprisingly, these disks are also misaligned with the orbit 


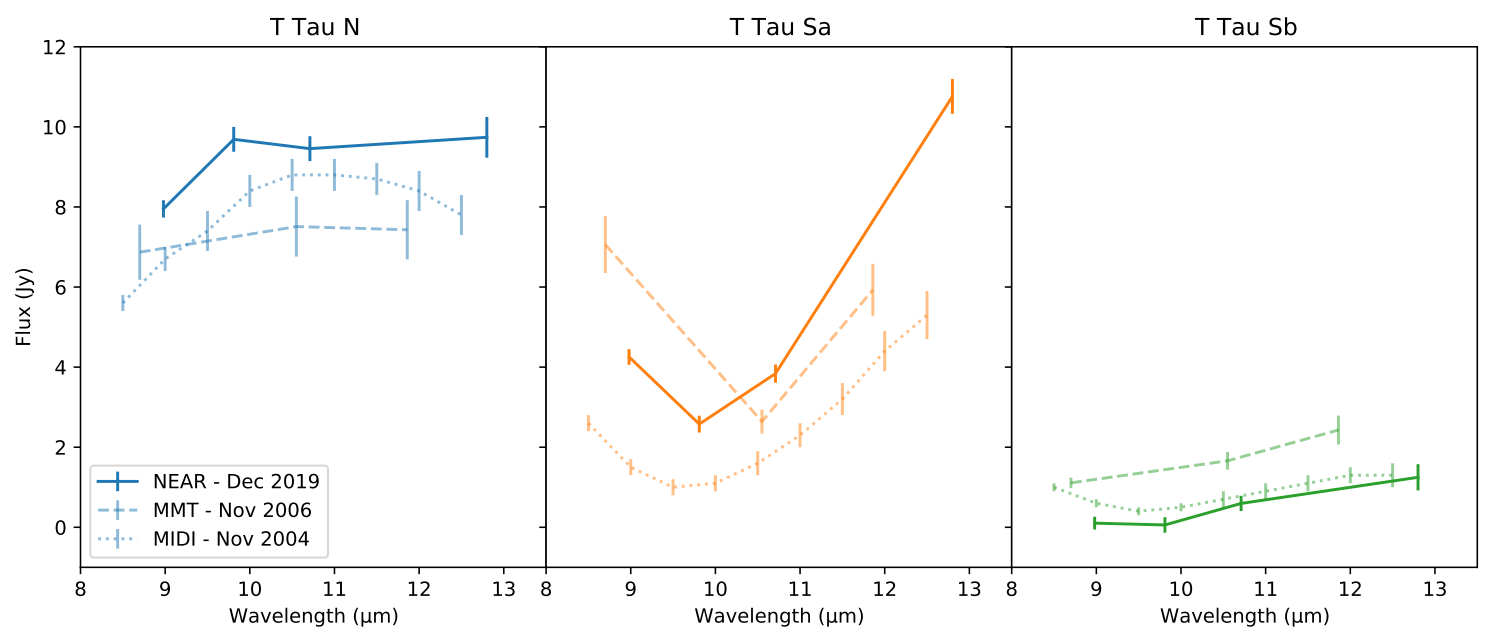

Fig. 7. Photometry of T Tau N (blue curves), Sa (orange curves), and $\mathrm{Sb}$ (green curves) from $\sim 8-13 \mu \mathrm{m}$. There are VISIR-NEAR data from December 2019 (solid curves, this work), MMT data from November 2006 (dashed curves, Skemer et al. 2008), and MIDI data from November 2004 (dotted curves, Ratzka et al. 2009).

of $\mathrm{T}$ Tau Sb around $\mathrm{Sa}$ and $\mathrm{T}$ Tau Sb's non-edge-on disk, regardless of their small physical separation of $\sim 15 \mathrm{au}$. At such small separations, tidal forces should align them within short timescales (Ratzka et al. 2009), challenging star-formation models and suggesting that multiple star formation can be turbulent (e.g., Whitworth 2001; Jensen et al. 2004).

At angular separations down to $\sim 0.2 \lambda / D$, we use kernel phase interferometry (Martinache 2010) together with the known positions of T Tau N, Sa, and Sb (Köhler et al. 2016) to robustly determine their brightnesses. We validate our methods by simulating and recovering mock data of the T Tau triple system using the observed PSF reference. We find that kernel phase interferometry, applied to high-Strehl images in the mid-infrared, is a powerful technique to achieve the highest possible angular resolution with single-dish telescopes. The adaptive optics-corrected VISIR-NEAR instrument provides a unique opportunity to perform such observations from the ground, while kernel phase will also find increased application in space-based observations with the James Webb Space Telescope (e.g., Ceau et al. 2019).

Acknowledgements. The NEAR project was made possible through contributions from the Breakthrough Foundation and Breakthrough Watch program, as well as through contributions from the European Southern Observatory. The manuscript was also substantially improved following helpful comments from an anonymous referee.

\section{References}

Artymowicz, P., \& Lubow, S. H. 1994, ApJ, 421, 651

Ceau, A., Mary, D., Greenbaum, A., et al. 2019, A\&A, 630, A120

Duchêne, G., Ghez, A. M., \& McCabe, C. 2002, ApJ, 568, 771

Duchêne, G., Ghez, A. M., McCabe, C., \& Ceccarelli, C. 2005, ApJ, 628, 832

Duchêne, G., Beust, H., Adjali, F., Konopacky, Q. M., \& Ghez, A. M. 2006, A\&A, 457, L9

Dyck, H. M., Simon, T., \& Zuckerman, B. 1982, ApJ, 255, L103
Foreman-Mackey, D. 2016, J. Open Source Softw., 1, 24

Foreman-Mackey, D., Hogg, D. W., Lang, D., \& Goodman, J. 2013, PASP, 125, 306

Ghez, A. M., Neugebauer, G., Gorham, P. W., et al. 1991, AJ, 102, 2066

Herbst, T. M., Beckwith, S. V. W., Glindemann, A., et al. 1996, AJ, 111, 2403

Ireland, M. J. 2013, MNRAS, 433, 1718

Jensen, E. L. N., Mathieu, R. D., Donar, A. X., \& Dullighan, A. 2004, ApJ, 600, 789

Kammerer, J., Ireland, M. J., Martinache, F., \& Girard, J. H. 2019, MNRAS, 486 639

Kasper, M. E., Feldt, M., Herbst, T. M., et al. 2002, ApJ, 568, 267

Kasper, M., Santhakumari, K. K. R., Herbst, T. M., \& Köhler, R. 2016, A\&A, 593, A50

Käufl, H.-U., Kasper, M., Arsenault, R., et al. 2018, in Proc. SPIE, SPIE Conf. Ser., 10702, 107020D

Köhler, R., \& Kubiak, K. 2020, Res. Notes Am. Astron. Soc., 4, 73

Köhler, R., Kasper, M., Herbst, T. M., Ratzka, T., \& Bertrang, G. H. M. 2016, A\&A, 587, A35

Koresko, C. D. 2000, ApJ, 531, L147

Kruegel, E., \& Siebenmorgen, R. 1994, A\&A, 288, 929

Lagage, P. O., Pel, J. W., Authier, M., et al. 2004, Messenger, 117, 12 Laugier, R., Martinache, F., Cvetojevic, N., et al. 2020, A\&A, 636, A21

Leisenring, J., Bary, J., \& Skrutskie, M. 2007, in American Astronomical Society Meeting Abstracts, Am. Astron. Soc. Meeting Abstr., 211, 62.12

Loinard, L., Torres, R. M., Mioduszewski, A. J., et al. 2007, ApJ, 671, 546

Martinache, F. 2010, ApJ, 724, 464

Martinache, F. 2013, PASP, 125, 422

Martinache, F., Ceau, A., Laugier, R., et al. 2020, A\&A, 636, A72

Mathis, J. S., Rumpl, W., \& Nordsieck, K. H. 1977, ApJ, 217, 425

Monnier, J. D., Tuthill, P. G., Ireland, M., et al. 2009, ApJ, 700, 491

Pope, B., Martinache, F., \& Tuthill, P. 2013, ApJ, 767, 110

Pope, B., Tuthill, P., Hinkley, S., et al. 2016, MNRAS, 455, 1647

Ratzka, T., Schegerer, A. A., Leinert, C., et al. 2009, A\&A, 502, 623

Sallum, S., \& Skemer, A. 2019, J. Astron. Telesc., Instrum. Syst., 5, 018001

Schaefer, G. H., Beck, T. L., Prato, L., \& Simon, M. 2020, AJ, 160, 35

Skemer, A. J., Close, L. M., Hinz, P. M., et al. 2008, ApJ, 676, 1082

Ströbele, S., Arsenault, R., Bacon, R., et al. 2006, in The ESO Adaptive Optics Facility, SPIE Conf. Ser., 6272, 62720B

van Boekel, R., Juhász, A., Henning, T., et al. 2010, A\&A, 517, A16

Whitworth, A. P. 2001, in The Formation of Binary Stars, eds. H. Zinnecker, \& R. Mathieu, IAU Symp., 200, 33

Yang, Y., Mayama, S., Hayashi, S. S., et al. 2018, ApJ, 861, 133 


\section{Appendix A: Tests with mock data}

Table A.1. Simulated and recovered relative fluxes for our mock data of the T Tau triple system.

\begin{tabular}{lccccccc}
\hline \hline Filter & Test no. & Sim. $f_{2}$ & Rec. $f_{2}$ (minimize) & Rec. $f_{2}$ (emcee) & Sim. $f_{3}$ & Rec. $f_{3}$ (minimize) & Rec. $f_{3}(\mathrm{emcee})$ \\
\hline \multirow{2}{*}{ ARIII } & 1 & 0.534 & 0.530 & $0.530_{-0.003}^{+0.003}$ & 0.012 & 0.009 & $0.010_{-0.002}^{+0.002}$ \\
& 2 & 0.273 & 0.271 & $0.271_{-0.002}^{+0.003}$ & 0.273 & 0.269 & $0.269_{-0.002}^{+0.002}$ \\
& 3 & 0.410 & 0.407 & $0.407_{-0.003}^{+0.003}$ & 0.137 & 0.133 & $0.133_{-0.002}^{+0.002}$ \\
& 4 & 0.491 & 0.488 & $0.488_{-0.003}^{+0.002}$ & 0.055 & 0.052 & $0.052_{-0.002}^{+0.002}$ \\
\hline SIV_1 & 1 & 0.273 & 0.267 & $0.254_{-0.014}^{+0.011}$ & 0.002 & 0.000 & $0.010_{-0.007}^{+0.011}$ \\
& 2 & 0.135 & 0.143 & $0.142_{-0.021}^{+0.021}$ & 0.135 & 0.121 & $0.122_{-0.017}^{+0.017}$ \\
& 3 & 0.202 & 0.208 & $0.208_{-0.023}^{+0.022}$ & 0.068 & 0.056 & $0.056_{-0.018}^{+0.018}$ \\
& 4 & 0.242 & 0.247 & $0.240_{-0.020}^{+0.018}$ & 0.027 & 0.017 & $0.023_{-0.014}^{+0.016}$ \\
\hline SIV_2 & 1 & 0.403 & 0.401 & $0.401_{-0.005}^{+0.005}$ & 0.066 & 0.064 & $0.064_{-0.005}^{+0.005}$ \\
& 2 & 0.232 & 0.232 & $0.232_{-0.005}^{+0.005}$ & 0.232 & 0.229 & $0.229_{-0.005}^{+0.005}$ \\
& 3 & 0.348 & 0.347 & $0.347_{-0.005}^{+0.005}$ & 0.116 & 0.113 & $0.114_{-0.006}^{+0.005}$ \\
& 4 & 0.418 & 0.416 & $0.416_{-0.005}^{+0.005}$ & 0.046 & 0.044 & $0.044_{-0.005}^{+0.006}$ \\
\hline NEII & 1 & 0.905 & 0.918 & $0.919_{-0.009}^{+0.009}$ & 0.116 & 0.140 & $0.141_{-0.0011}^{+0.012}$ \\
& 2 & 0.905 & 0.920 & $0.920_{-0.017}^{+0.019}$ & 0.500 & 0.535 & $0.535_{-0.027}^{+0.028}$ \\
& 3 & 0.905 & 0.920 & $0.921_{-0.011}^{+0.012}$ & 0.250 & 0.279 & $0.281_{-0.015}^{+0.015}$ \\
& 4 & 0.905 & 0.918 & $0.918_{-0.008}^{+0.009}$ & 0.100 & 0.124 & $0.124_{-0.011}^{+0.011}$ \\
\hline
\end{tabular}

Notes. The values of $f_{2}$ and $f_{3}$ correspond to T Tau Sa and Sb for the ARIII, SIV_1, and SIV_2 filters and to T Tau N and Sb for the NEII filter, respectively. We perform fits using both a Broyden-Fletcher-Goldfarb-Shanno least-squares algorithm (minimize) and an MCMC algorithm (emcee). 